\title{
La educación es un derecho, no es un privilegio
}

\section{Education is a right, not a privilege}

\section{DOI: $\underline{10.32870 / \text { dse.v0i19.611 }}$}

\section{Teresa González Luna Corvera*}

Educar es central en el desarrollo social, en la construcción de sociedades más justas y en el sostenimiento de la vida democrática. Se afirma que sin educación de calidad no será posible la democracia ni el crecimiento económico (Levinson y Berumen, 2007; Tedesco, 2012). Desde hace varias décadas se habla de la capacidad transformadora de la educación y de la urgencia de mejorar su calidad y asegurar la equidad para hacer frente a la pobreza y a las desigualdades sociales.

En las agendas del desarrollo sostenible y en el ámbito de la igualdad, la educación juega un papel decisivo: "la educación es la estrategia fundamental para avanzar en la cohesión y en la inclusión social" $y$, de la mano con el empleo, es uno de los ámbitos privilegiados de superación de los problemas sociales (OEI, 2010: 16); "la educación constituye hoy la palanca de la igualdad y un eslabón múltiple en el desarrollo" en América Latina (CEPAL, 2010). En este mismo sentido, los Objetivos para el Desarrollo Sostenible (ODS) plantean metas para el año 2030 que promueven la educación inclusiva de calidad, la igualdad de género, la reducción de la desigualdad y el fortalecimiento de la alianza mundial para el desarrollo sostenible (ODS, núms. 4, 5, 6 y 17).

La pregunta sobre los fines y los modos de la educación ha estado presente a lo largo de la historia en el pensamiento político y social moderno, en la que se destaca su papel fundamental en la construcción del Estado y de las sociedades, y ha generado múltiples respuestas desde las ciencias sociales e interpretaciones políticas controvertidas. El debate es permanente y se intensifica en coyunturas sociopolíticas específicas en las que está en juego la definición del curso de las políticas educativas nacionales. En las sociedades democráticas se da por sentado que la educación es un derecho humano fundamental, un bien social, un medio de acceso a otros bienes y oportunidades, una condición para la formación de la ciudadanía y la vida en democracia, un instrumento de cohesión social y proceso indispensable para la realización de las personas, más allá de valorar la educación como un factor clave para el mundo de la economía.

* Doctora en Estudios Científicos y Sociales. Profesora-investigadora en el Departamento de Estudios Políticos de la Universidad de Guadalajara. Líneas de investigación: derechos, ciudadanía y no discriminación. Coordinadora de la Cátedra UNESCO: "Igualdad y No Discriminación". México.teresa.gonzalez.luna@gmail.com 
En la actualidad, es amplia la legislación que define los contenidos y alcances del derecho a la educación, y prolífica la literatura que aborda el campo educativo. Se resalta su importancia radical y la relación de este con los demás derechos humanos, en la medida en que la educación potencia el desarrollo de las personas y es condición esencial para el disfrute de todos ellos. No se puede ejercer ninguno de los derechos civiles, políticos, sociales, económicos o culturales sin un mínimo de educación. ' En este sentido, para Bourdieu la educación lleva en sí el futuro, y en ella radica "la condición mayor de acceso al ejercicio de los derechos del ciudadano", con todo y las contradicciones y posibilidades de acción que conlleva (Bourdieu, 2008: 90).

Una parte significativa de los acuerdos internacionales y las políticas relacionadas con el derecho a la educación, derivados de la Declaración Universal de los Derechos Humanos (artículo 26), reconoce que la educación es un derecho humano fundamental e imprescindible para el ejercicio de todos los demás derechos, y asume que esta es un eje clave del desarrollo social. Dado su carácter vinculante, estos instrumentos son un parámetro de obligación para el Estado mexicano y se ha logrado priorizar la educación como un derecho social, alentando de manera puntual el acceso y la permanencia de la población en edad escolar en los servicios educativos del nivel básico.

El discurso de los derechos humanos, con sus insuficiencias y desafíos, se ha instalado desde hace tiempo y de distintos modos en la agenda pública internacional y nacional. Esta retórica ha ganado terreno, al mismo tiempo que las condiciones de existencia de la mayor parte de la población mexicana, la de los grupos sociales que de manera histórica y sistemática han sido discriminados, se deterioran aún más y se incrementan las brechas de desigualdad social. El hecho es que hoy la educación y la no discriminación son derechos incumplidos para millones de mexicanos.

En este complejo campo sobresalen las desigualdades estructurales que afectan a nuestra sociedad. Al contrastar la legislación vigente en materia de derechos humanos con los diagnósticos y datos disponibles sobre el sistema educativo nacional y las prácticas discriminatorias, aparecen de inmediato obstáculos y limitaciones de carácter estructural que impiden la concreción del derecho a la educación a los grupos de población discriminados.

La reciente Encuesta Nacional sobre Discriminación (Enadis, 2017) revela una distribución desigual de derechos, recursos y oportunidades en un país en el que se discrimina a las personas y grupos de población por razones diversas: el color de piel, la posición económica, la apariencia física, la edad, el origen étnico, la condición de discapacidad, el género, la identidad sexual, las creencias religiosas, entre otras. A pesar de su prohibición constitucional, se muestra que la discriminación está repartida de forma heterogénea a través de la geografía nacional, que persisten múltiples prejuicios, estereotipos y marcadores sociales en torno a las diferencias indi-

1 Por ejemplo, la libertad de expresión: ¿de qué sirve si la persona no tiene las capacidades de formarse un juicio personal y de comunicarlo? 0 el derecho al trabajo: ¿de qué sirve si se carece de las calificaciones necesarias para un buen trabajo? (Latapí, 2009: 258).

Diólo oos sobre Educación año 10 | número 19 | julio-diciembre 2019 | ISSN 2007-2171 
viduales e identidades grupales que alimentan la heterofobia (rechazo o diferente), la intolerancia y la exclusión, lo que deriva en la limitación o negación de derechos a quienes se considera diferentes.

Datos como los siguientes ilustran la forma en que la multiplicación de características e identidades susceptibles a la discriminación, como el género, la etnicidad, la discapacidad y otras, imponen barreras en el acceso al sistema educativo e incrementan las brechas de desigualdad entre las personas y grupos sociales: mientras que 3\% de la población total de personas de 15 a 59 años en México no sabe leer ni escribir, el analfabetismo alcanza $20.9 \%$ entre las personas con alguna discapacidad, $13.3 \%$ entre hablantes de lengua indígena, $6.9 \%$ entre la población que se identifica como indígena y $4.7 \%$ entre las personas afrodescendientes. ${ }^{2}$ En cada grupo discriminado, con excepción de la población con discapacidad — que no presenta diferencias de género-, las mujeres registran mayores porcentajes de población analfabeta que afecta a $16.1 \%$ de las mujeres hablantes de lengua indígena, a $8.4 \%$ de indígenas por adscripción, 5.8\% de afrodescendientes y 4.1\% de mujeres de la diversidad religiosa (Enadis 2017: 29-31).

Por otro lado, en el más reciente informe del Instituto Nacional de Evaluación Educativa (INEE, 2019: 16), se consigna que, si bien la cobertura en el nivel primaria que corresponde a las niñas, niños y adolescentes (NNA) de 6 a 11 años es prácticamente universal, a partir de los 12 años la tasa de asistencia disminuye, principalmente entre la población en condiciones de vulnerabilidad. En el caso de NNA en pobreza extrema, la tasa de asistencia pasa de $77.5 \%$ para aquellos de 12 a 14 años, a $48.4 \%$ para los de 15 a 17 años. Esta situación se agrava para NNA de localidades rurales en donde la inasistencia crece a $90.4 \%$ para el grupo de 12 a 14 , y a $63.2 \%$ para el de 15 a 17 años. Según datos de la Encuesta Intercensal, en 2015 casi 4.8 millones de NNA entre 3 y 17 años no asistía a la escuela; en comparación con las niñas (2.3 millones), son los niños quienes menos asisten a la escuela ( 2.5 millones). Para 2016 , solo $87.5 \%$ de la población de 17 a 21 años había concluido la educación básica. ${ }^{3}$

La ampliación de la cobertura y el aumento del acceso a la educación han sido dos objetivos centrales en los compromisos de desarrollo educativo nacional. A pesar de los notorios avances en la cobertura de la educación básica, son múltiples los problemas aún no resueltos: disparidades entre géneros, analfabetismo, abandono escolar temprano, trabajo infantil, bajo rendimiento académico, acceso limitado a la educación media y mucho más restringido a la educación superior, escasa calidad de la oferta educativa pública, y falta de pertinencia y adecuación de los programas curriculares de cara a la diversidad de poblaciones, circunstancias

2 Se considera analfabeta a una persona que no puede leer, escribir, ni comprender un texto corto sobre su vida cotidiana (analfabetismo absoluto), o por su incapacidad para utilizar sus destrezas de lectura, escritura y cálculo de forma eficiente en las situaciones habituales de la vida (analfabetismo funcional). De acuerdo con UNICEF (2000) se necesitan "no menos de cinco años de escolaridad para que una persona sea considerada realmente alfabetizada".

3 La cobertura educativa es tan solo uno de los indicadores que expresa hasta qué punto la sociedad garantiza la igualdad de oportunidades; ahora se plantea la inclusión educativa como uno de los retos, no solo técnico sino conceptual y político, para el desarrollo del país. 
y necesidades. Lo que se observa es que las diferencias en el acceso, progresión y conclusión educativa se profundizan y se amplían a lo largo de los niveles escolares y se reproduce la cadena de desigualdad en el mismo sistema educativo, afectando negativamente a los grupos discriminados en el ejercicio de su derecho a la educación.

Dada su naturaleza e implicaciones, la agenda educativa es compleja y se complica aún más en contextos de desigualdades sociales como el mexicano. Por un lado, hay que resolver los rezagos históricos acumulados y los problemas persistentes; por otro, se impone hacer frente a los actuales desafíos que plantea el acceso al conocimiento mediante el uso de las nuevas tecnologías de la información y las redes comunicacionales. En suma, para hacer efectivo el derecho a la educación en condiciones de igualdad, se requiere saldar deudas educativas históricas y presentes con los grupos discriminados que se encuentran en clara desventaja social frente a quienes se encuentran en situación de privilegio, pero también es necesario evitar que se acrecienten las desigualdades y se adicione la brecha digital entre grupos sociales y generaciones de población.

La educación y la discriminación son fenómenos socioculturales que afectan las condiciones de vida de las personas y determinan, de manera decisiva, la necesaria inclusión de todos los sectores y grupos en el desarrollo de la sociedad. En el análisis y deliberación sobre su estrecho nexo, pero sobre todo en el diseño e instrumentación de políticas públicas, es indispensable tener en cuenta que la cuestión social ha sido abordada en México a partir de dos fenómenos: la desigualdad y la pobreza desde la perspectiva del análisis económico, tanto en su vertiente liberal clásica o desde la perspectiva de la economía política (Fuentes, 2018). La mayor parte de los estudios se ha centrado en el análisis de las causas (nivel de ingresos) y en la distribución de diferentes recursos entre las personas y en cómo esta incide sobre resultados desiguales, dejando de lado otras condiciones sociales, políticas y culturales que generan desigualdades sociales.

A diferencia de la abundante e histórica literatura sobre educación desde diversas disciplinas científicas y perspectivas analíticas, los estudios teóricos y empíricos acerca de la discriminación son relativamente recientes, y su aparición en México coincide con su reconocimiento como problema público y el establecimiento de normas que la prohíben. La desigualdad de trato como realidad o fenómeno social, y la no discriminación como derecho, es un campo de estudio interdisciplinario en plena construcción, que cuenta ya con algunos diagnósticos y estudios empíricos - como el referido - sobre sus causas, modalidades, efectos e impactos, pero los significados del concepto, las categorías asociadas al mismo, los contenidos y alcances del derecho a la no discriminación y sus intersecciones con el ejercicio de otros derechos y circunstancias de la vida social son todavía motivo de investigación, discusión académica y deliberación política.

Diálo@os año 10 | número 19 | julio-diciembre 2019 | ISSN 2007-2171 


\section{El ejercicio del derecho a la educación sin discriminación}

La educación es un derecho humano fundamental que todos los Estados deben garantizar. El alcance de este derecho es amplio porque depende e incide en la cultura, en la situación económica y en las decisiones políticas, y también porque el ejercicio de este derecho incidirá en la vida social de las personas desde edades tempranas (INEE, 2019). En este sentido, se afirma que "no sería posible reparar el daño infligido a la persona en el curso de su vida por la falta de educación; quien carece de ella en su infancia y juventud queda por lo mismo excluido de la sociedad, expuesto a la pobreza y relegado en comparación con los demás ciudadanos" (Latapí, 2009: 258).

El derecho a la educación es universal, propio de toda persona, con independencia de su edad, género, lengua, origen social o étnico, condición de discapacidad, creencia religiosa, orientación sexual, preferencia política, posición social o cualquier otro atributo personal o grupal, y es obligación del Estado proporcionar educación a toda la población sin discriminación alguna. La educación es un derecho progresivo, en el sentido de que debe avanzar gradualmente para lograr su efectividad y no puede haber retroceso o disminución en su contenido, a la vez que es exigible y justiciable, toda vez que puede ser invocado ante los tribunales y ser objeto de medidas legislativas, políticas, administrativas o sociales, conducentes a hacer eficaz este derecho.

Sin embargo, hacer efectivo el ejercicio pleno de este derecho sin distinción alguna, en todos sus momentos y/o dimensiones (reconocimiento, protección, garantía y exigibilidad), bajo el principio de igualdad, en un contexto nacional de desigualdades y sistema de privilegios, recursos económicos estatales limitados y prácticas discriminatorias institucionalizadas, plantea serios dilemas éticos y problemas de aplicación de criterios de gestión a los gobiernos. Todo esto y más, es motivo de una intensa y polémica discusión pública que tiene como punto de partida la normatividad vigente respecto a los derechos humanos (incluidos los compromisos internacionales) y las alarmantes evidencias sobre una realidad excluyente en la educación, pero también, y de manera significativa y decisiva, están de por medio las históricas luchas de poder entre los actores que intervienen en los procesos educativos (gobierno, sindicatos, sociedad civil, padres de familia, academia, entre otros agentes). Con todo, no se puede obviar ni negar que ahora el debate sobre la educación y la igualdad se inscribe en el marco de los derechos humanos, y que resulta muy importante la contribución del pensamiento y la perspectiva antidiscriminatoria en y desde las ciencias sociales.

En México, la educación es un derecho fundamental consagrado en el artículo tercero constitucional, del que se desprende la Ley General de Educación, que regula la educación que imparte el Estado (federación, entidades federativas y municipios), sus organismos descentralizados y los particulares con autorización o reconocimiento de validez oficial de estudios. En sintonía con organismos internacionales (UNESCO, OCDE, CEPAL y UNICEF, entre otros), el gobierno mexicano y sus 
instituciones del sector educativo coinciden en que la educación sea universal, inclusiva y de calidad (INEE, 2019).

Conforme a estándares internacionales, la educación que proporcione el Estado en todas sus formas y niveles debe ser asequible, accesible, aceptable y adaptable. Estos cuatro elementos constituyen los componentes esenciales del derecho a una educación inclusiva. Se entiende por asequibilidad la obligación del Estado de garantizar la disponibilidad de escuelas, docentes, programas de enseñanza y material educativo, entre otros recursos. La accesibilidad involucra tres dimensiones: no discriminación, accesibilidad física y accesibilidad económica, y supone que todas las personas, particularmente las que viven en condiciones de vulnerabilidad, puedan participar en el sistema educativo. Por su parte, la aceptabilidad refiere a la obligación de asegurar que los programas de estudio y los métodos pedagógicos se ajusten a las normas mínimas establecidas en materia de enseñanza, y que estos sean aceptables tanto para los NNA como para los padres. Por último, la adaptabilidad implica una educación flexible que responda a las necesidades de las comunidades y de los estudiantes en contextos culturales y sociales diversos para garantizar, entre otras cuestiones, la permanencia de los NNA en el sistema educativo (Tomasevski, 2004).

No obstante que el derecho a la educación está en la base de las obligaciones del Estado y "es crecientemente reconocido como el punto de partida y el fundamento último de las políticas educativas de los Estados," la legislación mexicana no impone ninguna sanción a las autoridades que limiten o violen el derecho a la educación (Latapí, 2009: 256). Por otra parte, cabe advertir que, además del Estado, intervienen otros actores que también son responsables de satisfacer el derecho a la educación, lo que quiere decir que su realización depende de la confluencia de las políticas gubernamentales, en primer lugar, pero también de las acciones que emprende la sociedad en su conjunto (familias, fuerzas económicas, iglesias, organismos de la sociedad civil y medios de comunicación, entre otros).

Si bien queda claro en esta exposición que la educación no es una mercancía y el derecho a la educación no se limita a la prestación de un servicio público, lo cierto es que la educación oficial se ha valorado y legitimado como el camino más eficaz para lograr el desarrollo social, y en ella se depositan múltiples expectativas de cambio de la sociedad en sentido democrático. Se reconoce ampliamente, y con razón, que las escuelas son las únicas instituciones con capacidad y mandato para llegar a todas las personas de manera sistemática y directa, a la vez que representan el espacio fundamental de la socialización ciudadana y aprendizaje de lo común (González Luna, 2010).

En efecto, desde un punto de vista sociológico, se valora que la educación "es una parte o casi un sinónimo de socialización" $y$, a partir de la segunda mitad del siglo XX, el sistema educativo (la escolaridad) se considera como el "principal distribuidor y legitimador de las posiciones 
sociales diferenciadas de las personas" (Planas, 2018: 33-34). Así, a la cultura de privilegios, a la que hacen referencia diversos análisis sobre la discriminación, se suma la cultura instrumental y utilitarista que es característica de los sistemas educativos, particularmente de la escolarización. ${ }^{4}$ Lo cierto es que los cambios en la vida social, en particular los que tienen que ver con el mundo del trabajo, repercuten en el campo educativo (formal e informal) y hacen emerger nuevas racionalidades educativas, dentro y fuera del sistema escolar, lo que hay que gestionar. Como bien propone Planas (2018), se precisa pasar de la sociología del sistema escolar a la sociología de la educación que incorpore la diversidad de personas, racionalidades y métodos, pero también el riesgo. Se trata, pues, de dimensionar el derecho a la educación y considerar las distintas modalidades y tiempos en que se juega su reconocimiento y ejercicio, más allá de la escolarización.

En este orden de ideas, lo que hace que la educación escolarizada sea democrática es su concreción en un lugar y en un servicio en el que todas las personas encuentren el espacio para el desarrollo pleno de sus capacidades. En este contexto, la educación "es pública porque no excluye a nadie, y deja de serlo en el momento que segrega a los que no caben en determinados propósitos homogeneizadores que pretenden igualar a todas y todos con un alumnado-tipo que no existe en la realidad. Lo que existe es la diversidad y la diferencia. Existe entre el alumnado, como existe entre el profesorado" (Sansano, 2014: 39).

Ahora bien, en la medida en que la desigualdad de trato encuentra su fuerza en las representaciones simbólicas que los sujetos tienen sobre los grupos humanos, en los prejuicios y procesos de estigmatización de los "otros", la educación desempeña un papel crucial. Los hechos indican que la discriminación se aprende mediante los procesos de socialización temprana de los individuos que tiene lugar en las instituciones y en las prácticas informales, pero también en los procesos formales como la educación básica y el adoctrinamiento religioso (Rodríguez, 2011).

Desde esta perspectiva, la educación es el derecho más importante en la lucha contra la discriminación. Es el derecho social por excelencia, en la medida en que sintetiza de manera significativa las nociones modernas relacionadas con la libertad y la igualdad, además de que visibiliza y vigoriza el ejercicio de todos los derechos en su conjunto y de todas las personas sin distinción de ningún tipo.

Si bien el fenómeno de la discriminación en México ha estado presente a lo largo de los siglos, es hasta inicios del siglo XXI que aparece en la esfera pública como problema social y político de primer orden, y también como prohibición jurídica a partir del reconocimiento constitucional del derecho a la no discriminación. Este proceso de construcción del derecho antidis-

4 Jordi Planas (2018) encuentra que son escasos los estudios sobre la educación que no están referidos a los sistemas educativos, a la escolarización y a las instituciones escolares como objeto de estudio, muy de la mano de la teoría económica predominante de capital humano, que enfatiza la relación entre nivel educativo, salario y categoría profesional. Desde la perspectiva funcionalista, se ha planteado que el sistema escolar, dada su expansión, es un instrumento para la igualdad de oportunidades y el ascenso social con base en el mérito de las personas. En cambio, las teorías opuestas de matriz marxista interpretan que en el capitalismo la escuela funciona como un instrumento de legitimación y reproducción de las desigualdades de origen. 
criminatorio ha sido gradual y ha tenido que sortear dificultades de distinta índole, dando por resultado una definición legal:

queda prohibida toda discriminación motivada por origen étnico o nacional, el género, la edad, las discapacidades, la condición social, las condiciones de salud, la religión, las opiniones, las preferencias sexuales, el estado civil o cualquier otra que atente contra la dignidad humana y tenga por objeto anular o menoscabar los derechos y libertades de las personas" (artículo $1^{\circ}$ de la Constitución Política de los Estados Unidos Mexicanos). ${ }^{5}$

Sin duda, la estructura normativa de la sociedad es variable históricamente e influye en las ideas que se tienen acerca de los derechos humanos, la igualdad y la educación.

El derecho a la educación y el derecho a la no discriminación forman una pareja necesaria para la construcción de una sociedad democrática. Son derechos que comparten principios y se complementan bien en el plano discursivo y en las normas que los regulan y fundamentan, pero lo cierto es que su relación es difícil y no está exenta de dilemas y problemas que generan tensión a la hora de su realización, poniendo a prueba su efectividad. El contenido de ambos derechos es complejo, pero sobre todo el análisis de sus alcances, dada su estrecha conexión entre sí y con otros derechos humanos, en particular con los derechos sociales que son condición de posibilidad para su cumplimiento (Gutiérrez y Salazar, 2011).

Como puede constatarse en los criterios de las políticas educativas nacionales e internacionales, a los reclamos tradicionales de acceso a la educación se han sumado otras exigencias relacionadas con la inclusión de las diferencias y la calidad educativa para hacer efectivo el ejercicio pleno de este derecho. Durante las últimas décadas, en México se han realizado esfuerzos importantes por asegurar el acceso a la educación, mejorar la calidad, aumentar los años de la educación obligatoria, incrementar la cobertura, combatir el abandono escolar, mejorar la infraestructura, diseñar nuevos currículos y formar a los docentes, entre otros aspectos. Sin embargo, persisten graves problemas para lograr que la educación a la que todas las personas tienen derecho sea de calidad, basada en el respeto a las diferencias, en la libertad de las personas y en una pluralidad de posibilidades educativas que refleje la diversidad de las personas, aspiraciones y proyectos (INEE, 2019). Y es que igualdad se opone a desigualdad y no a la diversidad social.

5 En el caso mexicano, de acuerdo con Kaufman (2010), se define una "discriminación propia" legalmente tipificada, que describe la magnitud del fenómeno hacia determinados grupos sociales, al contrario de las definiciones imprecisas en las que todo trato diferente queda englobado en una "discriminación genérica", que desdibujan las situaciones jurídicamente condenables y dan lugar a posiciones demagógicas. En efecto, estos instrumentos jurídicos (tanto la cláusula antidiscriminatoria como la ley en la materia) han contribuido a visibilizar la discriminación y a imprimirle el carácter de problema público, así como a desmontar algunos de los prejuicios y estigmas normalizados para plantear nuevas concepciones de igualdad.

Diólopos 


\section{Inclusión frente a la exclusión: un dilema entre objetivos y desigualdades educativas}

Pobreza, desigualdad y exclusión son términos reveladores de una grave problemática de las sociedades contemporáneas, y se manifiestan claramente en el campo de la educación; han pasado a ser un lugar común en el discurso político, mediático y académico como "ruptura de lazo" que cuestiona la cohesión social. Derechos, desarrollo, igualdad e inclusión son algunos de los vocablos que expresan, en contraparte, la intención y los objetivos públicos de revertir y solucionar estos problemas sociales. Pero estos fenómenos estructurales no se reducen a la dimensión económica y es necesario incorporar en el debate, los estudios, el diseño de políticas públicas y en los mismos procesos educativos, los otros factores de orden cultural y educativo, social y político, incluso identitario, que son decisivos para entender la realidad y estar en condiciones de transformarla. Al admitir que las desigualdades y los desequilibrios sociales son también producidos culturalmente, surgen nuevos nudos conceptuales y prácticas problemáticas en los escenarios y políticas educativas actuales, que cabe considerar al abordar el vínculo entre los derechos a la educación y a la no discriminación, entre estos, y de manera especial: la tensión creciente inclusión/exclusión entre los derechos humanos que se afirman (reconocimiento, profundización y/o ampliación) frente a las fuerzas de la economía que se imponen, no ceden y tienden hacia la exclusión.

Al respecto, Autés señala que "la exclusión es un nombre puesto a los infortunios de la época, que dañan las articulaciones entre las diferentes esferas de la vida social, entre los diferentes universos, entre los diferentes mundos" y aparece como "imaginario de la caída social". En suma, la exclusión refiere a una desligadura entre la esfera económica y la política que se realiza en lo simbólico, y su resultado es la incapacidad para producir sentido y sujetos o actores. En este contexto, aparece la "inclusión" como nueva categoría de gestión que responde a este mal (la exclusión) y penetra las políticas públicas, planteando complejos problemas al Estado (Autés, 2004: 17 y 24). Uno de los riesgos, sobre los que advierte el autor y cabe reflexionar, es la pretensión de clasificar o categorizar a la población, que es de suyo heterogénea, y dar lugar a estatutos legitimados y estigmatizantes ("los pobres", "los discapacitados", "las mujeres", "Ios menores", "los migrantes" o "los indígenas", etc.) para estar en condiciones de lidiar con la diversidad y administrar desde el poder público las diferencias que le pueden resultar insoportables.

Las exclusiones son formas de discriminación directa o indirecta, intencional o por omisión, personal o institucional, situada o múltiple, reguladas por las leyes y/o normalizadas y legitimadas en prácticas culturales. No obstante, Castel se niega a llamar exclusión a todas las situaciones de desequilibrio social y alerta sobre el uso laxo, incluso peligroso, de esta noción que caracteriza a ciertas poblaciones que están o han estado al margen de la sociedad y da nombre a una multitud de situaciones que son dispares y en donde la especificidad de cada una queda diluida, imposibilitando análisis más penetrantes sobre las diferentes situaciones de las perso- 
nas y dando lugar a "tratar por separado ciertas situaciones límite que sólo adquieren sentido cuando se las inserta en determinados procesos. Los 'excluidos' están en la desembocadura de trayectorias, y de trayectorias diferentes. Nadie nace excluido, se hace" (Castel, 2004: 57).

Desde esta visión, referencias genéricas a la exclusión y a la desigualdad de trato en la educación pueden convertirse en una trampa para el pensamiento y el análisis de la realidad, dando lugar a políticas de inserción o inclusión coyunturales y focalizadas que sirven o pueden ser utilizadas para maquillar un supuesto entendimiento de estos fenómenos sociales. No se trata de prescindir de estas políticas que responden a grupos de población caracterizados en función de un problema o déficit específico y les atribuyen un estatuto especial -lo que parece necesario-, pero sí de advertir los riesgos y evitar que acrecienten o den lugar nuevas formas de discriminación.

Uno de los peligros es, al decir de Castel (2004), invisibilizar o desconocer el perfil y las circunstancias de los "nuevos públicos en dificultades" y sus diferencias irreductibles respecto a los grupos que, por el momento, entran en la tipología de población discriminada. ${ }^{6}$ Otro riesgo presente es que, en la medida en que el tratamiento social especial estigmatiza a las poblaciones implicadas, se pueden generar otras formas de discriminación basadas en las etiquetas asignadas oficialmente a ciertos grupos sociales, aunque el propósito de las políticas sea su inclusión (Castel, 2004, 69). Por otra parte, es necesario evitar las situaciones de "insertados permanentes" a los que nunca se logra integrar y luego son estigmatizados, y considerar que una cierta cantidad de personas se encuentran excluidas con carácter provisorio, pero en torno a su situación se instalan dispositivos que son, en cambio, permanentes o de duración indefinida. ${ }^{7}$

Ahora bien, el paradigma de desarrollo vigente en la región de América Latina, basado en los derechos humanos, plantea la generación de nuevas políticas que contribuyan a sostener y construir condiciones para la superación de las desigualdades sociales y la discriminación estructural. La CEPAL (2018) destaca el potencial del concepto de inclusión para el diseño de las políticas públicas encaminadas al desarrollo social, ya que permite abordar los efectos de la exclusión social como fenómeno concatenado, da profundidad al diseño e implementación de acciones orientadas a mejorar la calidad de vida de las personas y compromete a las instituciones.

6 Con referencia a la exclusión, Castel considera que las medidas tomadas para erradicarla cumplen la función de política social más general, con metas preventivas y no solo reparadoras. "Parece más fácil y realista intervenir sobre los problemas relativamente limitados que plantean los 'excluidos' [...] que controlar o intentar controlar los procesos desencadenantes de tal exclusión. Ocuparse de las consecuencias de estos procesos - o sea, ocuparse de los excluidos_- moviliza básicamente respuestas técnicas (aunque no sean fáciles de encontrar), pero el dominio del proceso exigiría un tratamiento político, en el sentido de la política global" (Castel, 2004: 61).

7 En este mismo sentido, Dovigo (2014) opina que el pensamiento basado en el etiquetado es un obstáculo para entender las dificultades que los estudiantes, incluyendo a los considerados "normales", tienen a lo largo de sus itinerarios educativos. El problema radica en la tendencia tradicional de las instituciones educativas a reconocer y comprender las situaciones problemáticas solo en términos de su clasificación en una categoría en particular, ya sea "discapacidad", "trastorno de conducta", "dificultades de aprendizaje", "orígenes culturales" u otras, lo que conduce a una perspectiva reduccionista, torna más difícil responder a situaciones con dificultades múltiples e impide identificar necesidades no clasificables en las tipologías existentes porque son nuevas, apartadas o atípicas.

Diólopos 
La noción de inclusión figura en los debates sobre desarrollo social y aparece de forma destacada en la Agenda 2030 para el Desarrollo Sostenible. Se trata de un concepto multidimensional que alude a diversos ámbitos de acción (social, económica, laboral y educativa, entre otras) y cuyo alcance y potencial no se ha definido con precisión. La CEPAL (2018) ha definido la inclusión como

La realización de los derechos, la participación en la vida social, el acceso a educación, salud y cuidado, así como a los servicios básicos de infraestructura, y la disponibilidad de recursos materiales como ingresos y vivienda. Remite a un proceso de mejoramiento de las condiciones económicas, sociales, culturales y políticas para la plena participación de las personas en la sociedad, que tiene tanto dimensiones objetivas como de percepciones (CEPAL, 2018: 16-17).

Otros organismos internacionales definen la inclusión social como el proceso de mejorar las formas de participación en la sociedad, en particular la de aquellas personas desfavorecidas por razones de edad, sexo, discapacidad, raza, etnicidad, origen, religión o condición socioeconómica, mediante mayores oportunidades, acceso a recursos, expresión de su voz y respeto de sus derechos.

En efecto, la inclusión en la educación es uno de los principales temas en el debate educativo contemporáneo. Su horizonte de referencia se ha ampliado gradualmente: al principio la inclusión se refería a la educación básica para las personas con discapacidad; ahora comprende todos los niveles educativos, incluyendo la educación superior, y a los grupos que por diferentes razones están en riesgo de cualquier tipo de exclusión (Dovigo, 2014). En consecuencia, la educación inclusiva se propone la eliminación de procesos excluyentes que se expresan en respuestas hacia lo diferente, lo otro distinto, y se justifica como un proyecto de lucha general contra el fracaso y la exclusión; un cambio cultural en la escolarización actual y futura; un compromiso político a favor de un mundo más justo (Azorín, 2017). Asimismo, a partir del principio de educación para todos, se considera que $a$ ) la dinamización de procesos inclusivos puede ser el medio más eficaz para combatir las prácticas y actitudes discriminadoras y excluyentes; $b$ ) la reestructuración de las instituciones educativas es necesaria para garantizar la debida atención a todos los estudiantes; y c) la exclusión escolar suele ser el preludio de otras trayectorias de exclusión (Gairín y Suárez, 2014).

La educación inclusiva enfrenta grandes retos, entre ellos la exclusión institucional que descansa en reglamentos, rituales, aparatos especializados y procedimientos, y que además aparece siempre justificada y legitimada, lo que no implica que sea justa, éticamente aceptable o políticamente defendible. Desde esta perspectiva, la exclusión aparece racionalizada por una maquinaria mental y consolidada por un dispositivo administrativo, institucional y jurídico, sin olvidar que el Estado es el que ostenta el punto de vista oficial y dominante (Castel, 2004: 82). 
Instaurar y difundir un discurso claro e inequívoco sobre el rechazo a la discriminación de cualquier tipo, es controvertido y genera resistencias. De acuerdo con Aguilar Villanueva (2005: 17), "en el campo de los derechos humanos, y específicamente de la igualdad y la antidiscriminación, se requiere un Estado activo, comprometido, promotor y facilitador, y no sólo un Estado abstencionista, que elude entrar en el terreno de los hechos sociales de la desigualdad, o un 'Estado gendarme', que interviene sólo cuando hay que sancionar notorias e intolerables infracciones". En tal sentido, se requiere de una directriz general que refleje la prioridad y voluntad política de los gobiernos para modificar la realidad discriminatoria, es decir, de políticas públicas que permitan al Estado garantizar los derechos humanos, vinculando las necesidades sociales de corto plazo con una visión política a mediano y largo plazo, para así eliminar inequidades (Aguilar Villanueva, 1993).

En suma, el enfoque de derechos humanos demanda un modelo de Estado que no es neutral ni ciego a las diferencias; por el contrario, desde su "función de protección efectiva" no puede quedarse sin actuar y debe tomar medidas igualitarias para proteger a los grupos de población que están en desventaja en el ejercicio de sus derechos. Al decir de Abramovich (2009: 8), "los derechos humanos conducen a la responsabilización del Estado, pero no dicen nada sobre cómo los estados garantizan estos derechos".

\section{Consideraciones finales}

La educación es un fenómeno sociocultural atravesado por múltiples determinaciones estructurales, que expresa las relaciones que se dan en el conjunto de la vida social y da lugar a un campo de estudio y acción específico. Su abordaje como derecho abre posibilidades para generar nuevos sentidos respecto a la relación entre la educación, el Estado y la sociedad, las políticas educativas y las obligaciones de los poderes públicos para asegurar el ejercicio pleno del derecho a la educación de todas las personas (Ruiz, 2012).

Desde las diferentes ciencias sociales y perspectivas analíticas que han trabajado y piensan sobre el fenómeno de la educación (historia, filosofía, antropología, sociología, economía, derecho, psicología, pedagogía y política), pero sobre todo en los recientes estudios específicos que lo abordan desde una perspectiva antidiscriminatoria, parece necesario creer genuinamente que la educación tiene valor en sí misma, más allá de la escolarización (educación formal), sin dejar de analizar el papel central que esta última tiene en los itinerarios educativos de las personas y en sus resultados sociales. Se precisa considerar los fuertes vínculos del sistema educativo con otras y nuevas modalidades educativas tradicionales (familias y comunidades) y no formales ni tradicionales (medios y tecnologías de la comunicación), que socializan y educan durante toda la vida, y que pueden asumir la diversidad de estudiantes y procesos educativos para incidir en el desarrollo inclusivo de las sociedades.

Diálo pos año 10 | número 19 | julio-diciembre 2019 | ISSN 2007-2171 
Por lo general, se buscan las causas de las desigualdades en los diferentes recursos y capacidades que tienen las personas, en las relaciones que tienen entre ellas y en las estructuras sociales. Si bien cada perspectiva arroja luz sobre un aspecto del fenómeno de la desigualdad social, se requiere conjugarlas e incorporar nuevos elementos para contar con un marco multidimensional que, además de analizar las variables económicas, identifique los factores de carácter político, social y cultural que intervienen, y considere los diferentes tipos de desigualdades (raciales, étnicas, etarias, de género, de clase, de identidad genérica, de creencias, etc.) sustentadas en prejuicios, estereotipos y estigmas discriminatorios (Reygadas, 2004). Se trata de explorar las relaciones entre el derecho antidiscriminatorio y el conjunto de derechos humanos, sobre todo en el análisis de sus contenidos y alcances dada su estrecha interdependencia. La comprensión de las relaciones entre los derechos humanos desde una perspectiva antidiscriminatoria, y sus cruces con y entre los problemas sociales, abre un campo de pensamiento y acción desde las políticas públicas.

Los derechos humanos generan, cuando están resguardados legalmente, oportunidades de actuar para exigir a los poderes públicos condiciones iguales y efectivas para su realización y alcanzar nuevos derechos. En este sentido, la lucha por un derecho sirve como enclave para la conquista de otros y, una vez reconocidos, los derechos no solo protegen, también dan poder a la ciudadanía. Sin embargo, hay que estar en alerta continua frente al riesgo de que el lenguaje de los derechos humanos se convierta en una distracción para no atender de fondo los problemas derivados de las desigualdades y mantener intocada la organización del poder y la economía. Se trata de ir más allá de la corrección política o de la "razón retórica", para evitar que en ella se encubran los estereotipos y prejuicios que nutren las prácticas discriminatorias (personales, sociales e institucionales) y se oculte el sistema de privilegios que impide el ejercicio efectivo de los derechos de todas las personas en condiciones de igualdad. Hemos visto que las normas constitucionales se han modificado muchas veces para modernizarse y ampliar la lista de derechos, entre ellos el derecho a la no discriminación, pero también advertimos que se preserva el orden político y económico establecidos.

Ante la amplia brecha existente entre las normas y su concreción, entre los derechos reconocidos y sus condiciones reales de efectividad, es evidente que no basta con las legislaciones que, aunque son necesarias, tienen valor simbólico y pueden generar u obligar a cambios institucionales, no son suficientes. Es necesario trabajar en el terreno de las convicciones y en la dimensión cultural mediante la educación para transformar desde fondo y apuntalar una cultura democrática incluyente. De manera particular, resulta crucial una política educativa encaminada a la construcción de sujetos con capacidad de agencia en un contexto de ejercicio y exigibilidad de derechos. 


\section{Referencias bibliográficas}

Abramovich, V. (2006). "Una aproximación al enfoque de derechos en las estrategias y políticas de desarrollo". Revista de la CEPAL, 88, 35-50. Chile: CEPAL.

(2009 ). "La situación de los derechos humanos en América Latina". Exposición realizada durante el panel "Derechos Humanos, Autoritarismo y Democracia. Los aprendizajes de la lucha por los derechos humanos para intervenir en los problemas del presente". Buenos Aires. ile:///C:/Users/teres/OneDrive/Documentos/Abramovich\%20 -\%20La\%20situaci\%C3\%B3n\%20de\%20los\%20DH\%20en\%20Am\%C3\%A9rica\%20Latina\%20-\%202009.pdf

Aguilar Villanueva, L. F. (2015). "Prólogo". En M. Del Pino Pacheco. Catálogo de medidas para la igualdad. México: Conapred.

(comp.) (1993). "Estudio introductorio". Problemas públicos y agenda de gobierno. México: Miguel Ángel Porrúa, 15-72.

Autés, M. (2004). "Tres formas de desligadura". En S. Karsz (coord.). La exclusión: bordeando sus fronteras. Definiciones y matices. Barcelona: Gedisa.

Azorín Abellán, C. M., P. Arnaiz Sánchez y J. J. Maquilón Sánchez (2017). "Revisión de instrumentos sobre atención a la diversidad para una educación inclusiva de calidad". Revista Mexicana de Investigación Educativa. http://www.redalyc.org/articulo. oa?id=14054387002

Bourdieu, P. (2008). Capital cultural, escuela y espacio social. Argentina: Siglo XXI Editores.

Castel, R. (2004). "Encuadre de la exclusión". En S. Karsz (coord.). La exclusión: bordeando sus fronteras. Definiciones y matices. Barcelona: Gedisa.

CEPAL (2018). Hacia una agenda regional de desarrollo inclusivo. Bases y propuesta inicial. Chile: Naciones Unidas, pp. 16-17.

(2010). "La hora de la igualdad. Brechas por cerrar, caminos por abrir". Documento del Trigésimo tercer periodo de sesiones, CEPAL, Chile. https://repositorio.cepal.org/ bitstream/handle/11362/2951/S2010988 es.pdf?sequence $=1$

Conapred (2018). Encuesta Nacional sobre Discriminación 2017 (Enadis). Prontuario de resultados. México: Conapred.

Dovigo, F. (2014). "El tratamiento de la diversidad en las instituciones educativas". En J. Gairín (coord.). Colectivos vulnerables en la universidad. Reflexiones y propuestas para la intervención. España: Wolters Kluwer.

Fuentes, M. L. (2018). Las muertes que no deben ser. Natalidad y mortalidad en México. México: Fondo de Cultura Económica.

Gairín, J. y C. I. Suárez (2014). "Clarificar e identificar los grupos vulnerables". En J. Gairín. Colectivos vulnerables en la universidad. Reflexiones y propuestas para la intervención. España: Wolters Kluwer. 
Gutiérrez Rivas, R. y P. Salazar Ugarte (2011). Igualdad, no discriminación y derechos sociales. Una vinculación virtuosa. México: Conapred.

González Luna, T. (2010). "Democracia y formación ciudadana". Cuadernos de Divulgación de la Cultura Democrática. México: IFE.

INEE (2019). "La educación obligatoria en México". Informe 2019. México: INEE. https://www.inee. edu.mx/wp-content/uploads/2019/04/P1/245.pdf

(2019). Marco referencial y metodológico para la evaluación de contenidos y métodos educativos. México: INEE.

Kaufman, G. A. (2010). Dignus interpares. Un análisis comparado del derecho antidiscriminatorio. Buenos Aires: Abeledo Perrot (Derecho Constitucional).

Latapí, P. (2009). "El derecho a la educación: Su alcance, exigibilidad y relevancia para la política educativa". Revista Mexicana de Investigación Educativa, 14. http://www.redalyc.org/articulo.oa?id=14004012

Levinson, B. y J. G. Berumen (2007). "Educación para una ciudadanía democrática en los países de América Latina: Una mirada crítica". Revista Electrónica Iberoamericana sobre Calidad, Eficacia y Cambio en Educación, 4, 16-31. http://www.rinace.net/arts/vol5num4/art1.pdf

OEl y CEPAL (2010). 2021 Metas educativas. La educación que queremos para la generación de los bicentenarios. España: OEI.

Planas, J. (2019). "Educación y sociología". En C. Palomar (coord.). Comprender la educación desde las ciencias sociales. México: Universidad de Guadalajara.

Reygadas, L. (2004). "Las redes de la desigualdad: un enfoque multidimensional". Política y Cultura, 22, 7-25. México: UAM-Xochimilco.

Rodríguez Zepeda, J. (2011). Democracia, educación y no discriminación. México: Cal y Arena.

Ruiz, M. (2012). "El retorno al derecho a la educación. Cosmopolitismo y agenciamiento". RMIE, 17(53), 351-359. México: COMIE.

Sansano, A. (2014). "Educación y colectivos vulnerables: una asignatura pendiente". En J. Gairín, G. Palmeros y Ávila, y A. Barrales Villegas (coords.). Universidad y colectivos vulnerables. Reflexiones y experiencias. México: Ediciones del Lirio, 31-44.

Tedesco, J. C. (2012). Educación y justicia social en América Latina. Argentina: FCE.

Tomasevski, K. (2005). "Indicadores del derecho a la educación". Revista del Instituto Interamericano de Derechos Humanos (IIDH), 40, 341-387. Costa Rica: IIDH. https://revistas-colaboracion. juridicas.unam.mx/index.php/rev-instituto-interamericano-dh/article/view/35957/32885 